La Revue

des Droits

de l'Homme

\section{La Revue des droits de l'homme}

Revue du Centre de recherches et d'études sur les droits fondamentaux

$10 \mid 2016$

Revue des droits de l'homme - $\mathrm{N}^{\circ} 10$

Entretien avec Guillaume Tusseau, Professeur de droit public à l'École de droit de Sciences Po, membre du groupe de travail pour l'avenir des institutions en 2015

\title{
Charlotte Girard
}

\section{(2) OpenEdition}

Journals

Édition électronique

URL : http://journals.openedition.org/revdh/2365

DOI : $10.4000 /$ revdh.2365

ISSN : 2264-119X

Éditeur

Centre de recherches et d'études sur les droits fondamentaux

Référence électronique

Charlotte Girard, «Entretien avec Guillaume Tusseau, Professeur de droit public à l'École de droit de Sciences Po, membre du groupe de travail pour l'avenir des institutions en 2015 », La Revue des droits de l'homme [En ligne], 10 | 2016, mis en ligne le 04 juillet 2016, consulté le 25 juillet 2020. URL : http:// journals.openedition.org/revdh/2365 ; DOI : https://doi.org/10.4000/revdh.2365

Ce document a été généré automatiquement le 25 juillet 2020.

Tous droits réservés 


\title{
Entretien avec Guillaume Tusseau, Professeur de droit public à l'École de droit de Sciences Po, membre du groupe de travail pour l'avenir des institutions en 2015
}

\author{
Charlotte Girard
}

VOUS AVEz PARTICIPÉ AU gROUPE de tRAVAIL SUR L'AVENIR DES INSTITUTIONS INSTALLÉ À l'assemblée nationale sous la présidence de claude bartolone, président de L'ASSEMBLÉE NATIONALE ET DE MICHEL WINOCK, HISTORIEN. UN PREMIER TOME DE PRÈS DE 1000 PAgES INTITULÉ « REFAIRE LA DÉMOCRATIE » EN EST ISSU LE 2 OCTOBRE DERNIER. APRÈs AVOIR RAPPELÉ LES gRANDES LIgNES DE LA CONVOCATION DE CE gROUPE, QUELLE APPRÉciation aVez-Vous de ce processus d'Élaboration? Est-ce bien DÉmocratique AU REgARd de L'AMBITION CONSTITUANTE QU'IL PORTE ?

S'agissant de la convocation, j'ai été sollicité comme constitutionnaliste avec l'optique ouverte que je n'étais pas un contributeur habituel aux débats constitutionnels en France, notamment dans les médias, les partis politiques ou au sein de précédents comités de réflexion. On a également fait valoir l'idée que le groupe réunirait non seulement des experts du droit et de la constitution - comme l'avait fait le Comité Balladur - mais également des chefs d'entreprise, des syndicalistes, des hommes et des femmes politiques, et parmi eux un philosophe et quelques constitutionnalistes. Cela m'a séduit et c'est pour cette raison que j'ai accepté. J'ai accepté de n'être pas la voix du droit, mais une voix parmi d'autres et parmi d'autres disciplines, de manière à entendre d'autres points de vue sur les institutions.

Peut-on parler d'un "groupe d'experts » ? Oui forcément, dans le sens où chacun est forcément expert de sa discipline ou de sa pratique professionnelle: philosophie, gouvernance d'entreprise, droits de l'homme, droit constitutionnel, syndicalisme, vie politique... On peut regretter qu'il n'y ait pas eu dans la commission 2 ou 3 citoyens 
tirés au sort. Cela aurait pu enrichir les débats, surtout si on part du présupposé qu'il n'y a pas besoin de « compétence » pour parler politique. Toutefois, il faut éviter une fallacie de composition: ce n'est pas parce qu'on est chacun expert dans son domaine, que le groupe est expert de quelque chose. L'ensemble du groupe n'était pas expert de la chose publique. Chacun a pu y réfléchir de manière très libre en discutant, en faisant valoir des points de vue qui n'étaient pas forcément les points de vue de sa discipline ou de sa pratique. Pour le dire de manière quelque peu imagée : chacun n'a pas cherché à jouer son propre rôle, et aucun n'avait été sollicité pour cela. Je n'ai pas parlé en tant que constitutionnaliste, professeur à Sciences Po, de tel âge et avec tel intérêt académique. Les autres non plus. Les discussions ont permis que l'on interagisse et que l'on apprenne. De ce point de vue là, j'ai trouvé l'expérience particulièrement réussie. La diversité des perspectives était intéressante. Un consensus a fini par émerger - du moins sur les problèmes majeurs. Sur les solutions, on peut être en désaccord, aussi bien sur telle ou telle solution ponctuelle que sur l'articulation de l'ensemble des solutions.

Concernant l'aspect démocratique du processus, on peut dire qu'il est en effet démocratique puisque l'initiative provient d'une institution qui est elle-même a priori qualifiable de "démocratique ", c'est-à-dire élue et représentative. Mais dans la mesure où ce n'est pas le peuple ou un jury citoyen qui a pris la décision, posé le diagnostic et formulé les propositions, il est toujours possible de considérer que l'entreprise demeure une discussion d'élites, et de dire que ce n'est pas démocratique. Je n'ai pas vraiment de jugement là-dessus.

\section{AVEZ-VOUS PARLÉ dU FAIT QUE VOUS ÉTIEZ EN TRAIN DE DISCUTER DE LA CONSTITUTION, DU « CONTRAT SOCIAL ", ET QUE DONC VOUS AVIEZ UNE AMBITION CONSTITUANTE ? EST-CE QUe L'idée d'une alternative en termes de procédure est VenUe dans le débat? AVEZ-VOUS ÉVOQUÉ LA POSSIBILITÉ QUE VOUS ÉTIEZ PEUT-ÊTRE EN TRAIN D'EFFECTUER UN TRAVAIL PRÉPARATOIRE À UNE ASSEMBLÉE CONSTITUANTE ?}

Il ne me semble pas que cela ait été le cas. En toute hypothèse, cela n'a pas été explicité de cette manière. En revanche, on a beaucoup centré nos analyses sur la réforme de la constitution : quand on a pensé institutions, on a pensé constitution et révision. Et pourtant, il n'était pas évident pour tous les participants que l'on devait rester dans le cadre de la Ve République.

Pour ma part, je pense que ce chiffrage est assez indifférent. Réformer la Ve pour faire une Ve bis ou se focaliser sur l'idole du numéro 6 ne me paraît pas très intéressant en soi. En revanche, il est intéressant de savoir, sur le plan historique, qui donne les numéros. Cette question m'avait été posée par un collègue canadien à l'occasion d'une recherche sur les cultures constitutionnelles comparées. Or je n'ai pas de réponse.

Chaque fois que l'on a pensé réforme des institutions, on a pensé inscription dans la constitution. Et quand on discutait notamment démocratie sociale, environnement, futur, prise en compte du long terme, c'était en termes d'inscription de ces notions dans la constitution. On n'a pas forcément eu conscience qu'il y a un parti pris làdedans, le parti pris selon lequel l'organisation de la société passe par un texte juridique qui s'appelle «constitution». Dans le même temps, il fallait bien reconnaître d'où l'on parlait. Si nous faisions valoir des idées, il fallait pouvoir les proposer au titre de la modification du texte constitutionnel, notamment afin d'en souligner l'importance. Mais il est également possible que cette forme de réflexe 
traduise au fond chez des «experts " qui ont des horizons très divers une nouvelle conception de la constitution, plus riche, plus substantielle et plus engagée idéologiquement, que l'on n'aurait pas conçue ainsi il y a 30 ans en France.

\section{EN QUOI EST-CE SI DIFFÉRENT DE CE QUI SE PASSAIT AVANT ? PAR EXEMPLE, LA SOLUTION À LA CRISe ALgérienne a Été PRÉsentée en 1958 comme passant PAR Un Changement DE CONSTITUTION?}

Oui c'est vrai. C'est même une caractéristique récurrente de la culture française : tout changement d'ère politique et sociale est compris comme devant passer par un changement constitutionnel. C'est l'héritage de 1789. Mais ce qui est nouveau, c'est que l'on veuille enrichir le contenu de la constitution, élargir la constitution à de nouveaux objets. D'une certaine manière, cela conduit peut-être à vous donner raison : il s'agit d'une façon de renouer avec la richesse du contenu des constitutions formelles révolutionnaires, dont les dispositions dépassaient très largement ce que l'on inclut traditionnellement sous le terme de « constitution matérielle ».

LE RAPPORT DU gROUPE DE TRAVAIL NE LIVRE PAS UNE SOLUTION CLÉ EN MAINS MAIS UN AVIS DONT LES OPINIONS QUI ONT CONTRIBUÉ À L'ÉLABORER SONT VISIBLES ; UNE SORTE DE SYSTÈMES D'OPINIONS SÉPARÉES (LES CONTRIBUTIONS DES MEMBRES SONT FORMULÉES), Y COMPRIS DES OPINIONS DISSIDENTES. QUE PENSEZ-VOUS DE CE SYSTÈME d'ÉLABORATION DE LA POSITION DU gROUPE DE TRAVAIL, NOTAMMENT EN TERMES D'EFFECTIVITÉ ?

C'est en effet là un point important. Quant au consensus, on n'a pas été forcés à agréer un ensemble de propositions ou de thèses. C'est bien un système d'opinions séparées, encore que je ne sois pas sûr que cela forme "système ». Je trouve que c'est très bien et je regrette vraiment, en raison de mes fonctions au sein du Conseil supérieur de la magistrature, d'avoir dû observer une certaine réserve et de n'avoir pas pu rédiger une contribution propre. C'était l'une des manières de tirer avantage de ce groupe de discussion et de sa composition. Personne n'attendait qu'on propose à l'issue de nos travaux 10 réformes de la constitution avec 10 nouvelles rédactions d'articles qui seraient immédiatement intégrées à un projet de loi constitutionnelle. Nous étions donc très libres, parce que nous n'étions pas directement contraints par une visée opérationnelle immédiate. Ensuite, le fait que l'on puisse tous exprimer notre vision et notre réflexion postérieure aux débats de la commission, c'est l'intérêt de la chose. Le rapport en effet n'est pas du tout un ensemble de solutions mais un ensemble de problèmes, de regards sur des problèmes, et donc un ensemble de manières de construire les difficultés institutionnelles, de les expliciter, de les identifier. Parce qu'on est à peu près tous d'accord pour considérer qu'il y a un problème. Il y a une série de manières d'imaginer précisément les contours de ce problème et d'en inférer des solutions et quelques perspectives.

Quant à l'effectivité, on peut considérer que le travail, que toutes nos heures de réflexion et de discussion, sont cette chose maintenant livrée au débat public. Aux associations, aux hommes politiques, aux partis, aux militants, aux collègues, etc. de s'en saisir, de le critiquer, de le commenter. On peut considérer que c'est effectif immédiatement par le fait de livrer ces visions du problème au débat public, parce que l'on n'a pas de visée opérationnelle. On espère que certaines choses changent. Mais cela reste vague et on n'aboutit pas à une position très articulée. C'est une limite de notre travail. Mais en même temps, on ne devait pas trop viser à fournir les futurs éléments du programme d'un candidat à l'élection présidentielle. Nous sommes tous convaincus que la constitution sera au cœur ou un point important du programme des futurs candidats. Parce que d'une part, il le faut: le genre littéraire d'un 
programme présidentiel veut qu'il propose des changements institutionnels. Parce que d'autre part, il y a l'évidence certaines de difficultés de la démocratie française actuelle. Mais on partageait une mise en garde contre l'idée - qui aurait pu être contradictoire - que l'on était peut-être en train d'écrire le canevas possible d'un programme présidentiel, d'abord parce que l'initiative venait de l'Assemblée - ce qui est exceptionnel sous la $\mathrm{Ve}$ - et ensuite parce que la présidentialisation de la Ve République est en soi un des éléments de difficulté. Si notre proposition, constatant certains des inconvénients de la présidentialisation, avait ambitionné de figurer dans le programme d'un candidat à la présidence, attendu comme sauveur constitutionnel, cela aurait été d'une certaine manière self-defeating.

Donc l'effectivité de notre travail est dans le fait de donner un instrument pour le débat. On peut trouver aujourd'hui qu'elle est limitée. Depuis qu'il est sorti, en effet, le rapport n'a pas eu beaucoup d'écho. On peut attribuer cela à plusieurs facteurs, dont le premier est me semble-t-il que l'on estime qu'il y a toujours plus important que les institutions et leur réforme dans le contexte actuel. Ceci me parait parfaitement légitime et naturel: on peut tous comprendre sans la moindre démagogie que les personnes qui sont les plus en souffrance aujourd'hui sur le plan personnel, familial, économique, social, etc. estiment avoir plus urgent à penser que la réforme des institutions.

PARMI LeS ÉlÉMENTS QUI FONT dOUTER de L'EFFECTIVITÉ OU QUI NE PERMETTENT PAS DE percevoir l'effectivité du travall de ce groupe, je pensais au destin du QUESTIONNAIRE QUI A ÉTÉ MIS EN LIgNe SUR tOUTES LES PROPOSITIONS QUI ONT ÉTÉ

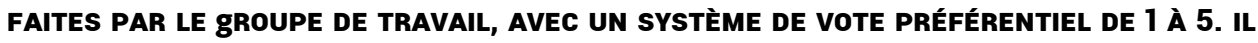
RÉVÈle UNE VOLONTÉ RÉELLE d'INNOVATION. VOUS AVEZ DONC IMAgINÉ DES MOYENS DE DIFFUSER LA RÉFLEXION EN VOUS SOUMETTANT VOUS-MÊMES À CE QUESTIONNAIRE, EN EN PUBLIANT LES RÉSULTATS ditS « AgRÉgÉS " ET EN LE METTANT EN LIgNE TEL UN « QUIZZ ". POUR AUTANT, ON N'EN A PAS FAIT gRAND-CHOSE. N'Y AURAIT PAS LÀ QU'UN EXERCice de STYLE ?

Je ne crois pas. Il y a une volonté très sincère de réfléchir à ces questions. On peut être encore plus sévère et dire que c'est une mystification pour enterrer les questions les plus importantes. Mais il me semble que ce serait injuste. Pour préparer ce questionnaire, nous avons eu toute liberté pour ajouter des questions, changer des formulations, imaginer d'autres hypothèses de solutions. Personne n'a été piégé par ce questionnaire. Il n'y a pas eu de "guidage » complètement contraignant de ce qu'on pouvait répondre.

À titre personnel, ce questionnaire m'a permis de clarifier mes propres prises de positions sur un certain nombre de questions que je me posais ou que je ne me posais pas. La méthode avec 5 points me permettait de préciser mon degré d'engagement, en termes idéologique ou en termes de fonctionnalité des institutions, envers telle ou telle proposition. Le petit manque - très difficile à combler au demeurant - est de faire en sorte d'expliciter des choix, non pas sur chaque item de réforme, mais sur les effets systémiques des items de réformes, par exemple le quinquennat avec réforme du Sénat-CESE, ce n'est pas pareil que le quinquennat tout seul, de même que le septennat sec, ce n'est pas pareil que le quinquennat avec inversion du calendrier et réforme du Parlement, facilitation du référendum, et éventuellement du recall, ou pas. J'imagine qu'il y a des techniques à inventer. Cela manquait peut-être parce qu'à mesure que je répondais à certaines questions, j'étais conduit à me poser la question de savoir si en mettant 5 à telle réponse, c'était 5 à condition que j'aie mis 5 
également à telle autre réforme hypothétique, ou si les deux variables étaient totalement indépendantes. Et si je "ratais» la première réforme que j'aurais souhaitée, cela aurait donné un résultat complètement opposé dans l'autre item. La construction de «second best » a peut-être été un peu compliquée par cette méthode.

ChAQUE THÈME COMPORTE UNE PARTIE diAgNOSTIC TRÈs LUCIDE SUR LE MALAISE DANS LES INSTITUTIONS DE LA VE RÉPUBLIQUe POINTANT tOUS LES DÉFAUTS DE L'ÉQUiLIBRE

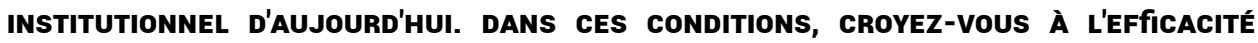
D'UNE RÉFORME INSTITUTIONNELLE QUI NE REMETTRAIT PAS EN CAUSE LA CONSTITUTION DE 1958 MAIS SE CONTENTERAIT DE RÉVISER LE DISPOSITIF EXISTANT SANS RÉÉCRIRE L'ENSEMBLE DU TEXTE ?

Je suis assez sceptique sur la symbolique de la 6e République qui ferait changer d'âge par rapport à la Ve. Mais c'est aussi un scepticisme qui est lié au fait que je ne crois pas que cela changerait la vie des citoyens de "passer à une 6e République ». Peutêtre est-ce une manière implicite de dire qu'un rafistolage aussi large soit-il suffirait pour que tout aille mieux. Peut-être qu'un changement important du texte irait de pair avec un énorme débat citoyen qui serait nécessaire pour satisfaire les besoins de l'ensemble de la population. C'est très prospectif et cela suppose que l'engagement populaire et une réécriture totale de la Ve vers la 6e, ne soient pas instrumentalisés ou phagocytés par certains groupes d'intérêts ou certains relais d'opinions classiques. Il était normal quand on a commencé à travailler dans le comité qu'on ne nous dise pas qu'un choix était fait d'emblée pour la Ve ou pour la 6e - chacun ayant son opinion tout au long du spectre, la mienne étant l'indifférence quant à la numérotation. Rester dans la Ve ou affecter de rester dans la Ve peut être une stratégie consistant à proposer des changements offensifs sans avoir l'air de réclamer un basculement vers une 6e, bref de les faire passer sans avoir l'air de tout remettre en cause. À l'inverse, instaurer en grande pompe une nouvelle constitution qui serait substantiellement la même que la précédente aurait tout d'un artifice mystificateur.

SUR LE FOND, LE RAPPORT FAIT HABILEMENT UNE PLACE DE CHOIX AUX DROITS DU PEUPLE EN VISANT LA RÉHABILITATION dU RÉfÉRENDUM. AU REgARd DES débATS QUI ONT AgITÉ LE groupe de travall à ce suJet, diriez-vous que cette visée tend À réparer une INJUSTICE OU À REMOdELER PROFONDÉMENT LES INSTITUTIONS ?

Réparer une injustice, c'est difficile à dire parce que la Ve est celle qui restaure le référendum qui avait quand même mauvaise presse au regard de l'histoire constitutionnelle française. Cette dernière opinion a d'ailleurs eu l'occasion de s'exprimer au sein du groupe. Remodeler profondément les institutions? Je ne sais pas si c'était l'ambition de l'ensemble du groupe, mais ce serait la mienne. Il faut toutefois préciser les contraintes institutionnelles que l'on fait peser sur l'exercice du référendum, les processus de rappel (révocation), les initiatives populaires, etc., en termes de majorités, d'ampleur de la participation, de modalités de recueil des soutiens, etc. Mais mettons que ces initiatives soient sincèrement possibles avec des délais raisonnables, un nombre de signatures préalables qui puissent être recueillies par des processus assez simples, par voie électronique, et pas $45 \%$ de la population pour soumettre une proposition, alors, dans ces conditions, cela peut être une option radicale, qui change la Ve et donne tout son sens à l'article 3 relatif à l'exercice de la souveraineté par la voie des représentants et par la voie du référendum. L'épouvantail en la matière, c'est l'article 11 réformé qui n'est qu'un simili référendum populaire. Il faut un référendum qui produise des effets soit institutionnels par des destitutions, soit normatifs par la mise en vigueur de normes 
juridiques de rang important, législatif ou constituant, si l'on veut développer une participation directe en matière constituante.

Il y avait toutefois des méfiances dans la commission. Les propositions sont donc endessous de ce que j'aurais espéré mais c'est le fruit d'un débat et il me semble qu'il était difficile d'obtenir quelque chose de plus offensif.

L'INSTITUTION PRÉSIDENTIELle CONCENTRE LES PLUS gRANDS POUVOIRS DONC LES PLUS gRANDES PRÉoccupations. Le RAPPORT PROPOSE NOTAMMENT LE SEPTENNAT NON

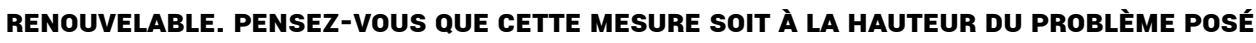
PAR LE STATUT CONSTITUTIONNEL DU CHEF DE L'ÉTAT ? LA QUESTION DE LA SUPPRESSION De L'INSTitution PRÉsidentielle - AUtrement dit L'OPTION RADicale - A-T-elle Été POSÉE?

Pour autant que je me rappelle, l'hypothèse de la suppression de l'institution présidentielle n'a pas été formulée. Mais se pose à nous la question préalable du positionnement en tant que membre de la commission : propose-t-on les solutions les plus hardies ou propose-t-on les solutions urgentes et immédiatement faisables aujourd'hui, ou encore propose-t-on ce que les partenaires institutionnels sont susceptibles d'accepter, qui se situerait entre notre idéal absolu et le simplement urgent et impératif? Une autre difficulté tenait au fait que si un groupe provenant de l'Assemblée proposait d'emblée la suppression de l'institution présidentielle, cela pourrait sembler un genre de conflit institutionnel mal venu ou inconvenant. En même temps cela aurait donné davantage de publicité à notre rapport !

Est-ce que le septennat non renouvelable est la solution à tout? Non évidemment. Le problème est comment on connecte cette proposition avec les autres réformes: le septennat non renouvelable? Oui ou non, mais avec quelle durée pour le Parlement, avec quel type de limitation de la succession des mandats dans le temps pour le Parlement? Avec quel type de Parlement, bicaméral ou monocaméral, avec quel bicamérisme renouvelé, etc. ? Le tout est complexifié par la difficulté d'identifier des relations de causalité très déterminées et très assurées entre une réforme et tel ou tel effet institutionnel. Il en est resté que tout en étant à peu près tous d'accord pour dire que le quinquennat et la présidentialisation dont il est à la fois l'effet et la cause sont un des éléments du problème, on a quand même du mal à imaginer quelque chose de radicalement différent et je ne crois pas qu'on se soit beaucoup prononcé pour la fin de l'élection au suffrage universel direct du Président, certains pour des raisons d'attachement à cette modalité, d'autres se disant que c'est le moment principal où le peuple discute politique, s'engage dans un débat et participe. À l'encontre de cet argument, d'autres ont pu dire que ce "débat public » est faussé parce qu'il ne porte que sur l'homme ou la femme providentiels pour être au bout du compte déçu trois mois après et faire enrager un peu plus, faire craquer un peu plus la société. C'est donc aussi un problème. Le rapport propose une évolution un peu technique, d'ampleur un peu réduite, qui mériterait d'être très expliquée, surtout dans l'économie d'ensemble de la réforme du régime. Donc, pour répondre directement : non, à soi seul, ce n'est pas à la hauteur du problème posé par le statut constitutionnel du chef de l'État. Mais justement ce ne doit pas être lu à soi seul.

QUANT AU PARLEMENT, IL APPARAît SYSTÉmATIQUEMENT COMME LE " gRAND BRÔLÉ " DE La Ve RÉpublique. mais SI les mesures proposées SONt louables, elles Ne SEMBLENT PAS NON PLUS À LA HAUTEUR du MAL ET CELA EST MANIFESTE, PARFOIS MÊME AVOUÉ, dans le rapport. pourquol n'aVolr pas afFronté plus franchement le PRoblème en REMettant en CAUSe l'économie générale des rapports entre 


\begin{abstract}
POUVOIR EXÉcutif ET POUVOIR LÉgISLATIF? PARCE QU'ON VOIT MAL COMMENT LA PROPOSITION QUI CONSISTE À PERMETTRE AU PARLEMENT d'ÊTRE MIEUX INFORMÉ des DÉcIsIONS EUROPÉENNES EN COURS POURRAIT LUI DONNER PLUS DE POUVOIR DANS LE RAPPORT QUI L'OPPOSE AU POUVOIR EXÉCUTIF.
\end{abstract}

C'est sans doute vrai. Mais peut-être est-ce une bonne idée au regard du compromis que David Cameron vient de négocier à Bruxelles aux termes duquel, dorénavant, si un certain nombre de Parlements sont tous en désaccord avec une mesure, il peut y avoir au moins un certain changement, une discussion plus approfondie. C'est donc peut-être « gadget » de revaloriser le Parlement sur ce point, mais si le Parlement est le « grand brûlé » de la Ve République, l'Europe n'est pas non plus correctement prise en compte dans la constitution.

Pourtant, je ne suis pas totalement d'accord avec le fait de dire que les propositions ne sont pas à la hauteur. On peut toujours vouloir plus et mieux. Mais justement sur ce sujet, on a entendu des choses assez offensives et déroutantes. Par exemple, tout ce que Bruno Latour a dit sur le «Parlement des choses", sur le Parlement du temps long, j'ai trouvé que c'était assez osé. Cela brusquait l'imaginaire de la Ve et ce qu'on pouvait partager. De même, la critique de la manière dont l'exécutif organise les rapports d'information et de production législative de la Haute administration avec le Parlement. On a toujours ce que l'on peut considérer comme la «tarte à la crème " s'agissant de l'inflation législative, des lois mal rédigées, de l'accablement du Parlement, les sessions extraordinaires, l'urgence, etc. Mais le fait d'en discuter avec les députés qui parlent de leur expérience personnelle, du rythme de travail, de l'absence de réflexion approfondie, alors même qu'ils préféreraient avoir le temps de détailler certaines mesures ou d'en discuter beaucoup plus, et de manière multipartisane, avec des échanges riches, m'a fait prendre conscience que ce n'était pas qu'un problème littéraire ou quantitatif. Pour les représentants, qui pour beaucoup d'entre eux veulent bien faire leur travail au profit de l'ensemble de la population, cela n'a pas qu'un effet macro sur le plan institutionnel, mais un effet personnel sur le rapport de représentation et la discussion de la loi qui est vraiment problématique. Il me semble donc que l'on n'est pas si timide que cela sur le Parlement. Quant à tout ce qui est proposé sur le Sénat ou le CESE, j'ai trouvé des choses assez radicales et nouvelles, notamment sur la reconquête du temps long, autrement dit le temps de la délibération. Le consensus qui se formait sur un certain nombre de modifications assez substantielles m'a semblé plus ferme que ce que j'aurais pu imaginer. Dans notre réflexion, il y a aussi quelque chose qui pesait en arrière-plan : le Parlement que va faire émerger la réforme du non-cumul si elle est préservée. Le Parlement de 2017 ne sera pas comme les autres et cela va renouveler un certain nombre de catégories dans le personnel politique avec des effets induits assez importants - si on laisse à la réforme le temps de produire ses effets. Si l'on adoptait en plus un non-cumul dans le temps des mandats, par exemple 2 ou 3 pas plus par individu pour l'ensemble de sa vie politique, cela aurait un effet sur le renouvellement des personnes, mais aussi des idées, des perspectives portées, le renouvellement du débat, donc. Ça peut sembler une mesure anodine, qui n'est pas forcément intégrée dans la constitution, mais qui pourrait avoir de très grands effets institutionnels.

QUANT À LA JUSTICE, AU MOMENT OÙ L'ÉTAT D'URgENCE CONDUIT À FAIRE BASCULER LA COMPÉTENCE EN MATIÈRE DE LIBERTÉ INDIVIDUELLE DE L'ORDRE JUDICIAIRE VERS L'ORDRE ADMINISTRATIF, LE SILENCE DU gROUPE SUR LE DUALISME JURIDICTIONNEL - LE JUgE 


\section{ADMINISTRATIF - ET L'INDÉPENDANCE DE LA JUSTICE VOUS SEMBLE-T-IL RÉTROSPECTIVEMENT UN ACTE MANQUÉ ?}

Je ne m'explique pas bien cette absence. Peut-être est-ce parce qu'on est parti du texte existant qui ne mentionne pas la justice administrative. Donc on n'a pas imaginé de réfléchir de manière très concrète à la question.

\section{ENCORE UN IMPENSÉ...}

Oui enfin c'est une hypothèse. On n'a pas réfléchi outre mesure à la réforme de la Justice administrative. Peut-être aussi est-on très satisfaits dans le groupe de la manière dont fonctionne la Justice administrative.

\section{AUTEUR}

\section{CHARLOTTE GIRARD}

Maîtresse de conférences à l'Université de Paris Ouest Nanterre la Défense 